\title{
Aberrant Forehead Sensory Nerves Found in Forehead Lift Surgery
}

\author{
Molaei $\mathbf{H}^{1,2 *}$, Taheri $\mathbf{A}^{1}$, Hajebi $\mathbf{R}^{3}$, Rahmati J ${ }^{1}$ and Mahmoodi $\mathbf{M}^{1}$ \\ ${ }^{1}$ Department of plastic \& reconstructive surgery, Tehran University of Medical Sciences, Iran \\ ${ }^{2}$ Department of plastic \& reconstructive surgery, Sina Hospital, Tehran University of Medical \\ Sciences, Tehran, Iran \\ ${ }^{3}$ Department of general surgery, Tehran University of Medical Sciences, Tehran, Iran
}

\section{Clinical Note \\ Volume 5 Issue 1}

Received Date: January 11, 2021

Published Date: February 15, 2021

DOI: $10.23880 /$ ijtps-16000154

*Corresponding author: Hojjat Molaei, Department of plastic \& reconstructive surgery, Tehran University of Medical Sciences, Vali-e-asr Hospital, Imam Khomeini Hospital Complex (IKHC), Bagherkhan st., Towhid Sq., Tehran, Iran, Tel: +989127798804; Email: hmolaei@sina.tums.ac.ir

\section{Clinical Note}

Forehead contains sensory innervations primarily composed of supraorbital, supratrochlear and zygomaticotemporal nerves on each side. These cutaneous nerves branch all wide the forehead and have their anatomical importance. They can be entrapped between muscles and induce headaches, which is the foundation of migraine surgery. On the other hand, during facial rejuvenation it is vital to know their anatomical routes to prevent iatrogenic injuries. Finally, during facial traumas, they are predisposed to lacerations and contusions. So, enough knowledge about these anatomical targets is mandatory.

Numerous studies notified these routes according to anatomical landmarks, and highlighted to keep in mind during surgical and nonsurgical procedures. Supraorbital nerve exits are considered by authors like Saylam, et al. who evaluated 500 samples and analyzed fronal and supraorbital foramen and notches. Accordingly, they found average distances from the midline to the frontal foramen (notch) and the supraorbital foramen (notch) were $20.24 \mathrm{~mm}$ and $25.23 \mathrm{~mm}$, respectively $[1,2]$.

Nanayakkara, et al. (2018) tried to demonstrate supraorbital nerve exits from 58 dry intact adult skulls in a Sri Lankan population to determine precise position in relation to the facial landmarks to suggest safe regional anesthesia, and to avoid iatrogenic nerve injuries during surgery of the orbito-facial region. They illustrated supraorbital nerve exits were mostly as notches (73.8\%) and the rest as foramina (26.2\%). Accessory exits were seen in $18.9 \%$ skulls. However, Ibrahim et al (2018) in a study on the postmortem CT scan of 104 Malaysian crania, revealed combinations of supraorbital foramen and notches, bilaterally between ancestors (Malay, Chinese, and Indian). They revealed bilateral supraorbital notch was the most prevalent combination [3]. Among these studies and similar articles, we found combination of foramen and notches for supraorbital exits with approximately defined distances from midline and orbital rim. But we did not found extra nerves besides original supraorbital nerves. Here, we present a 60 year- old female who admitted for forehead rejuvenation and brow lift. She had experiences with headaches of vertex and forehead under medical treatment. But, she did not receive nerve block. We did open forehead lift with hairline incision. During flap elevation we found 2 significant aberrant nerves with 2-3 $\mathrm{mm}$ diameter ( $40 \mathrm{~mm}$ away from midline and 12 $\mathrm{mm}$ above orbital rim, bilaterally) (Figure 1).

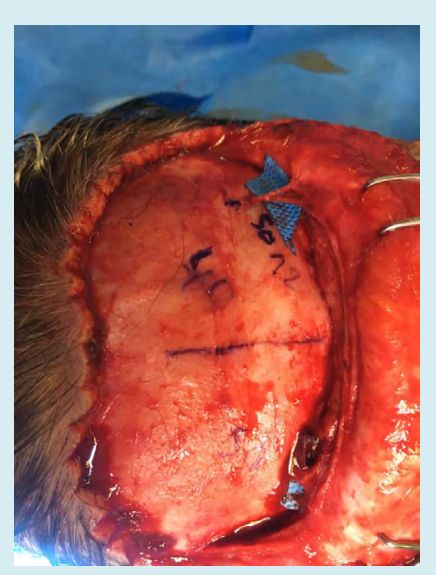

Figure 1: Aberrant sensory forehead nerves above normal supraorbital and supratrochlear nerves $12 \mathrm{~mm}$ above orbital rim and $40 \mathrm{~mm}$ lateral to midline bilaterally. 


\section{International Journal of Transplantation \& Plastic Surgery}

Though, the main supraorbital nerves were found exiting from their notches as usual during orbital rim release and muscle cut. We only freed nerves and removed compression of muscle. Aesthetic results were pleasant, but patient did not report pain relief despite nerve release, maybe due to aberrant nerves. This finding wants to remind the importance of glabellar nerves which can assume as trigger points of headaches to be treated [4], while, extra anatomical nerves, can change the results of nerve block during regional anesthesia and migraine surgery.

\section{References}

1. Saylam C, Ozer MA, Ozek C, Gurler T (2003) Anatomical variations of the frontal and supraorbital transcranial passages. The Journal of Craniofacial Surgery 14(1): 10-
12.

2. Nanayakkara D, Manawaratne R, Sampath H, Vadysinghe A, Peiris R (2018) Supraorbital nerve exits: positional variations and localization relative to surgical landmarks. Anatomy Cell Biology 51(1): 19-24.

3. Ibrahim A, Attalla SM, Alias A, Swarhib M, Abu Bakar SN, et al. (2019) Osteometric Analysis of Supraorbital Foramen And Notch In Malaysian Crania. Asian J Pharm Clin Res 11(10): 347.

4. Ortiz R, Gfrerer L, Hansdorfer MA, Nealon KP, Lans J, et al. (2020) Migraine Surgery at the Frontal Trigger Site: An Analysis of Intraoperative Anatomy. Plastic reconstructive surgery 145(2): 523-530. 